\title{
Trastornos de personalidad en los cónyuges de pacientes con dolor crónico
}

\author{
E. Collazo, M.A. Aragonés y D. Muñoz \\ Clínica del Dolor. Hospital Universitario Reina Sofía. Córdoba
}

Collazo E, Aragonés MA, Muñoz D. Trastornos de personalidad en los cónyuges de pacientes con dolor crónico. Rev Soc Esp Dolor 2014; 21(5): 235-241.

\begin{abstract}
Background and objectives: It has been detected determined models of performance in the consorts of patients with chronic pain, especially with fibromyalgia (FM), raising the question exist some shots or personality disorders in these consorts? If the answer were yes, would be important know if it produces this characteristic in the consort in a concrete time of the pain evolution.

Subjects and methods: Epidemiological observational descriptive cross-sectional study that consisted in requesting to the partners of patients with chronic pain (90 subjects) derived to the Pain Clinic that fill in the Multifactorial Personality Questionnaire HERAS and the Personality Questionnaire CEP.

A group of 60 consorts of patients without chronic pain was included as a control.

Results: The analysis of the questionnaires confirmed the predominance of concrete personality disorders in the consorts and its association with some conditions like time of coexistence and total value of the pain scale, as this like the predominance of determined shots of personality, especially in consorts of patient with FM.

Conclusions: It seems that a fast, simple and reliable detection of the features and especially the personality disorders of the consorts of patients with chronic pain, especially fibromyalgia, so try to avoid the deterioration of family life of these patients.
\end{abstract}

Key words: Chronic pain. Fibromyalgia. Consorts. Personality disorders.

Recibido: 09-05-13.

Aceptado: 15-08-13.

\section{RESUMEN}

Introducción y objetivos: se han detectado determinados modelos de actuación en los cónyuges de pacientes con dolor crónico, especialmente con fibromialgia (FM), surgiendo la pregunta ¿existen ciertos rasgos o trastornos de personalidad en estos cónyuges? Si así sucediera, sería importante averiguar si se produce esta característica en el cónyuge en un momento concreto de la evolución del dolor.

Pacientes y métodos: estudio epidemiológico observacional descriptivo transversal que consistió en solicitar a las parejas de pacientes con dolor crónico (90 sujetos) derivadas a la Clínica del Dolor que cumplimentaran el Cuestionario Multifactorial de Personalidad HERAS y el Cuestionario de Personalidad CEP. Como control se incluyó un grupo de 60 cónyuges de pacientes sin dolor crónico.

Resultados: el análisis de los cuestionarios confirmó la predominancia de trastornos concretos de personalidad en los cónyuges y su asociación con ciertas condiciones como el tiempo de convivencia y el valor total de la escala de dolor, así como la predominancia de determinados rasgos de personalidad, especialmente en cónyuges de pacientes con FM.

Conclusiones: parece conveniente aplicar un método rápido, sencillo y fiable de detección de los rasgos y especialmente de los trastornos de personalidad de los cónyuges de pacientes con dolor crónico, principalmente con FM, con el fin de intentar evitar el deterioro de la convivencia familiar de estos pacientes.

Palabras clave: Dolor crónico. Fibromialgia. Cónyuges. Trastornos de personalidad.

\section{INTRODUCCIÓN}

El dolor crónico figura entre los principales motivos de consulta médica $(1,2)$. Parece que algunos factores psicosociales, como el apoyo social percibido, influyen de 


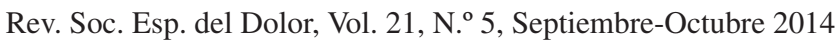

manera favorable en la percepción del dolor (3), contribuyendo al bienestar subjetivo y al estado de salud funcional en pacientes con desórdenes reumáticos crónicos $(4,5)$. En consecuencia, el apoyo social parece contribuir a la disminución del dolor y de los índices de depresión y de ansiedad.

Los trastornos psicológicos y los trastornos funcionales o la sensibilización central forman parte del círculo de la fibromialgia (FM); se complementan y se autoalimentan (6). Para encontrar soluciones hay que romper por algún lado este círculo vicioso, ya que hasta ahora carecemos de una terapéutica realmente efectiva, y este lado podría ser el psicológico.

Los índices de depresión se correlacionan de manera negativa con el apoyo percibido en el entorno próximo, el cual incluye al cónyuge, a la familia y al círculo de amistades. Por su parte, los índices de ansiedad muestran una correlación negativa débil con el apoyo percibido desde el entorno familiar y social, pero no con el apoyo percibido de la figura del cónyuge $(7,8)$.

Poco se ha escrito sobre los cónyuges de los pacientes que padecen dolor crónico, especialmente FM, excepto lo importantes que pueden y deben ser para esa persona; es como si de ellos dependiera la curación o mejoría de los pacientes, asumiendo en ocasiones un rol que son capaces de llevar a cabo gracias a sus perfiles psicológicos. El cónyuge puede pensar que el dolor crónico podría ser causa de un trauma en la vida del paciente, sintiéndose responsable de ese trauma en alguna medida.

En un estudio (7) la figura de la pareja, con una puntuación media de 5,86 \pm 0,22 puntos (IC del $95 \%, 5,424$ 6,288 ), fue valorada de forma positiva por aproximadamente 1 de cada 2 sujetos encuestados (48,9\%), aunque es considerable la proporción de personas $(26,2 \%)$ que valoró esta misma fuente de apoyo de manera negativa. La valoración media del apoyo recibido desde el núcleo familiar fue de 5,14 \pm 0,19 puntos (IC del $95 \%, 4,762-5,517$ ), con una distribución homogénea de las puntuaciones. Por el contrario, destaca el elevado porcentaje de personas $(49,3 \%)$ que puntuó de manera negativa $(3,79 \pm 0,17$; IC del $95 \%, 3,457-4,124)$ el apoyo recibido desde su entorno social próximo.

Algunos autores utilizan el término burn-out familiar (9) para identificar las frecuentes quejas victimistas, muy repetidas por pacientes con fibromialgia, presentando relatos que describen cuotas muy altas de sobrecarga psicológica.

Detectamos intuitivamente un determinado modelo de actuación en los cónyuges de pacientes con dolor crónico, especialmente con FM, surgiendo la pregunta ¿existe un determinado tipo de personalidad en estos cónyuges? La cronicidad del dolor suele conducir a quien lo padece a un estado de impotencia y desesperación; cabe preguntarse si sucede algo parecido en el cónyuge o si hay cambios en sus rasgos psicológicos o en su personalidad con el paso del tiempo, produciéndose una especie de agotamiento por la exposición a un estímulo permanente. Si así sucediera, sería importante averiguar si este cambio en el cónyuge se produce en un momento concreto de la evolución del dolor.

Se ha encontrado escasa bibliografía que permita responder a las preguntas planteadas previamente, por lo que nos propusimos realizar un estudio sobre este terreno. El objetivo principal del presente trabajo ha sido determinar, a través de cuestionarios autoaplicados, si existen rasgos y trastornos de personalidad en los cónyuges de pacientes con dolor crónico en general y con FM en particular. Si así fuera, pretendíamos averiguar si existen factores con los que se asocian especialmente.

\section{PACIENTES Y MÉTODOS}

Se trata de un estudio epidemiológico observacional descriptivo transversal realizado en la Clínica del Dolor del Hospital Universitario Reina Sofía, de Córdoba, desde julio de 2010 hasta julio de 2012.

A los cónyuges de pacientes con dolor crónico que fueron derivados sucesivamente a una consulta concreta de la Clínica del Dolor se les solicitó que cumplimentaran el Cuestionario Multifactorial de Personalidad HERAS (10) y el Cuestionario de Personalidad CEP (11), ambos autoaplicados, hasta completar el número de la muestra; se escogieron estos cuestionarios por su fácil cumplimentación y corrección. Previamente se explicó el estudio a los cónyuges y firmaron el documento de consentimiento informado específico para participar en él. Escogimos arbitrariamente un tamaño muestral de 90 sujetos (60 FM: 30 dolor crónico) al no haber encontrado estudios anteriores sobre este tema. Dentro del grupo de pacientes con FM se incluyeron exclusivamente los cónyuges de los que tuvieran confirmada la enfermedad por el Servicio de Reumatología. El único criterio de exclusión considerado fue que el cónyuge no supiera leer ni escribir. Se registraron las negativas a participar. Como control para valorar los rasgos y trastornos de personalidad en la población se incluyó un grupo de 60 cónyuges de pacientes sin dolor crónico que acudieron a otras consultas del hospital.

Las variables de interés fueron las indicativas de rasgos (inestable, introvertido, agresivo, indeciso, inseguro, sumiso) y trastornos de personalidad (antisocial, bordeline, ciclotímica, dependiente, evasiva, hipocondríaca, paranoide, de conversión, inmadura, esquizoide, explosiva, narcisista, esquizotípica, obsesiva).

Se consideraron potencialmente predictoras las siguientes variables: total de la escala del dolor, cronicidad del dolor, tiempo transcurrido desde el diagnóstico de FM del paciente, puntuación del Cuestionario de Impacto de Fibromialgia (CIF) (12) y años de convivencia. 
Los pacientes derivados a la Clínica del Dolor llevan años padeciendo la enfermedad, por lo que fue importante

TABLA I. ESCALA COMPLETA DE VALORACIÓN DEL DOLOR DE LOS PACIENTES

\begin{tabular}{|c|c|}
\hline \multicolumn{2}{|c|}{ Intensidad subjetiva } \\
\hline Sin dolor & 0 \\
\hline Ligero & 1 \\
\hline Molesto & 2 \\
\hline Intenso & 3 \\
\hline Insoportable & 4 \\
\hline \multicolumn{2}{|c|}{ Frecuencia } \\
\hline Nunca & 0 \\
\hline Rara vez & 1 \\
\hline Frecuente & 2 \\
\hline Muy frecuente & 3 \\
\hline Continúo & 4 \\
\hline \multicolumn{2}{|c|}{ Consumo de analgésicos } \\
\hline Ninguno & 0 \\
\hline Ocasional & 1 \\
\hline Regular & 2 \\
\hline Mucho & 3 \\
\hline Muchísimo & 4 \\
\hline \multicolumn{2}{|c|}{ Incapacidad } \\
\hline Ninguna & 0 \\
\hline Ligera & 1 \\
\hline Moderada & 2 \\
\hline Ayuda & 3 \\
\hline Total & 4 \\
\hline \multicolumn{2}{|l|}{ Sueño } \\
\hline Normal & 0 \\
\hline Despierta a veces & 1 \\
\hline Despierta 1 vez & 2 \\
\hline Despierta más de 1 vez & 3 \\
\hline Insomnio & 4 \\
\hline
\end{tabular}

valorar el tiempo de evolución del dolor. Otras variables fueron edad, sexo, estudios, estado de salud y ocupación del cónyuge; las dos últimas podrían constituir factores de confusión.

La escala habitual de valoración del dolor (Tabla I) presenta la ventaja de medir varias variables distintas de forma que, en conjunto, representan la característica de interés, siendo un índice que al combinar varias mediciones mejora la precisión con la que se valora el alivio del dolor.

Se utilizó el programa SPSS para realizar estadística descriptiva (tendencia central, frecuencia), prueba de Chicuadrado para variables dicotómicas (incluida la prueba exacta de Fisher cuando fue necesaria) y t de Student para las cuantitativas. Se obtuvo la V de Cramer para comparar con la odds ratio, especialmente cuando la aplicación de esta medida no era apropiada. En la elaboración de este proyecto se han seguido las recomendaciones de la guía STROBE (13).

Se respetaron los principios éticos recogidos en la Declaración de Helsinki y se obtuvo el informe favorable de la Comisión de Investigación del Hospital. Toda la información obtenida esta sujeta al mismo nivel de confidencialidad que la contenida en la historia clínica (Ley 41/2002, Ley 15/1999, R.D. 994/99).

\section{RESULTADOS}

Se reclutaron 90 cónyuges (91,1\% varones) de pacientes con dolor crónico. Un 60 \% manifestaron no padecer enfermedad alguna. Solo dos cónyuges se negaron a participar en el estudio. En la tabla II se especifican los datos referentes a la edad del cónyuge, duración del dolor (cronicidad) en el paciente, total de la escala del dolor en el paciente, tiempo transcurrido desde el diagnóstico en los pacientes con FM, puntuación CIF y años de convivencia.

En la tabla III se muestra la frecuencia de los rasgos y trastornos de personalidad de los cónyuges. No se encontraron sujetos con personalidades de tipo antisocial, borderline, evasiva, dependiente, agresiva ni esquizotípica entre los cónyuges de pacientes con dolor crónico no FM. En el conjunto, destacan un 21,6\% de cónyuges con trastorno de conversión, un $25 \%$

TABLA II. ESTADÍSTICA DESCRIPTIVA GENERAL

\begin{tabular}{lccccc}
\hline & $n$ & Mínimo & Máximo & Media & Desv. típ. \\
\hline Edad cónyuge (años) & 90 & 26 & 81 & 51,68 & 9,716 \\
Duración del dolor paciente (años) & 88 & 1 & 35 & 12,05 & 9,193 \\
Tiempo del diagnóstico de FM (años) & 59 & 1 & 16 & 3,83 & 3,519 \\
Puntuación CIF paciente & 51 & 39 & 96 & 69,10 & 13,034 \\
Total escala dolor paciente & 89 & 7 & 20 & 14,30 & 2,940 \\
Años de convivencia & 90 & 1 & 50 & 26,20 & 10,175 \\
\hline
\end{tabular}


Rev. Soc. Esp. del Dolor, Vol. 21, N. ${ }^{\circ}$ 5, Septiembre-Octubre 2014

TABLA III. FRECUENCIA DE LOS TRASTORNOS Y RASGOS DE PERSONALIDAD DE LOS CÓNYUGES

\begin{tabular}{cccccccc}
\hline $\begin{array}{c}\text { Personalidad } \\
\text { y rasgos }\end{array}$ & $\begin{array}{c}\text { Total cónyuges } \\
\text { control }\end{array}$ & $\begin{array}{c}\text { Total cónyuges } \\
\text { estudio }\end{array}$ & $\begin{array}{c}\text { De pacientes } \\
\text { FM }\end{array}$ & $\begin{array}{c}\text { De pacientes } \\
\text { no FM }\end{array}$ & V de Cramer & OR & Sign. \\
\hline Antisocial & $2(3,3 \%)$ & $2(2,3 \%)$ & $2(100 \%)$ & 0 & 0,11 & 58 & 0,45 \\
Ciclotímica & $1(1,6 \%)$ & $7(8 \%)$ & $4(57,1 \%)$ & $3(42,9 \%)$ & 0,06 & 0,63 & 0,42 \\
Dependiente & $1(1,6 \%)$ & $3(3,4 \%)$ & $3(100 \%)$ & 0 & 0,13 & 87 & 0,29 \\
Evasiva & $0(0 \%)$ & $5(5,7 \%)$ & $5(100 \%)$ & 0 & 0,17 & 145 & 0,13 \\
Hipocondríaca & $1(1,6 \%)$ & $10(11,4 \%)$ & $8(80 \%)$ & $2(20 \%)$ & 0,99 & 2,11 & 0,29 \\
Bordeline & $1(1,6 \%)$ & $2(2,3 \%)$ & $2(100 \%)$ & 0 & 0,11 & 58 & 0,44 \\
Paranoide & $1(1,6 \%)$ & $13(14,8 \%)$ & $9(69,2 \%)$ & $4(30,8 \%)$ & 0,02 & 1,12 & 0,56 \\
Conversión & $6(10 \%)$ & $19(21,6 \%)$ & $14(73,7 \%)$ & $5(26,3 \%)$ & 0,07 & 1,49 & 0,17 \\
Inmadura & $3(5 \%)$ & $4(4,5 \%)$ & $3(75 \%)$ & $1(25 \%)$ & 0,03 & 1,50 & 0,59 \\
Esquizoide & $3(5 \%)$ & $6(6,8 \%)$ & $5(83,3 \%)$ & $1(16,7 \%)$ & 0,09 & 2,59 & 0,35 \\
Explosiva & $5(8,3 \%)$ & $5(5,7 \%)$ & $3(60 \%)$ & $2(40 \%)$ & 0,03 & 0,72 & 0,53 \\
Narcisista & $1(1,6 \%)$ & $14(15,9 \%)$ & $11(78,6 \%)$ & $3(21,4 \%)$ & 0,10 & 1,98 & 0,25 \\
Esquizotípica & $2(3,3 \%)$ & $1(1,1 \%)$ & $1(100 \%)$ & 0 & 0,07 & 29 & 0,67 \\
Obsesiva & $1(1,6 \%)$ & $22(25 \%)$ & $17(77,3 \%)$ & $5(22,7 \%)$ & 0,12 & 1,94 & 0,84 \\
Inestable & $3(5 \%)$ & $52(57,8 \%)$ & $34(65,4 \%)$ & $18(34,6 \%)$ & 0,03 & 0,87 & 0,006 \\
Introvertida & $8(13,3 \%)$ & $77(85,6 \%)$ & $53(68,8 \%)$ & $24(31,2 \%)$ & 0,11 & 1,89 & 0,22 \\
Agresiva & $1(1,6 \%)$ & $11(12,2 \%)$ & $8(72,7 \%)$ & $3(27,3 \%)$ & 0,04 & 1,38 & 0,46 \\
Indecisa & $4(6,6 \%)$ & $21(23,3 \%)$ & $13(61,9 \%)$ & $8(38,1 \%)$ & 0,05 & 0,76 & 0,07 \\
Sumisa & $5(8,3 \%)$ & $79(87,8 \%)$ & $52(65,8 \%)$ & $27(34,2 \%)$ & 0,04 & 0,72 & 0,46 \\
Insegura & $9(15 \%)$ & $21(23,3 \%)$ & $13(61,9 \%)$ & $8(38,1 \%)$ & 0,05 & 0,76 & 0,07 \\
\hline OR Ora
\end{tabular}

OR: Odds ratio.

con personalidad obsesiva, un $57,8 \%$ con inestabilidad emocional, un $85,6 \%$ de introvertidos, un $87,8 \%$ con personalidad débil o sumisa y un 23,3\% de indecisos. Se encontraron diferencias significativas $(\mathrm{p}<0,05)$ entre los cónyuges del grupo control y del grupo de dolor crónico en cuanto a la prevalencia respecto a los tipos ciclotímico, hipocondríaco, paranoide, narcisista, agresivo e indeciso; aunque las diferencias aumentaron $(\mathrm{p}<0,01)$ en el tipo obsesivo y se intensificaron $(\mathrm{p}<0,001)$ en los tipos inestable, introvertido y sumiso.

El número y frecuencia de los rasgos y trastornos de personalidad agrupados que presenta cada cónyuge del grupo de estudio se muestran en la tabla IV. Al comparar el grupo de los cónyuges de pacientes con FM con el de los cónyuges de pacientes con dolor crónico no FM no se encontraron diferencias significativas entre las diversas variables estudiadas, excepto en lo relativo al valor total de la escala de dolor $(14,95 \pm 2,6$ vs. $13,03 \pm 3$; IC $95 \%$ $=0,6$ a 3,$1 ; \mathrm{t}$ Student $=3,0 ; \mathrm{p}=0,003)$ y a la inestabilidad emocional (34 de 52: 65,4\% vs. 18 de 52: 34,6\%; $\mathrm{p}=$ $0,006)$. En lo relativo a la indecisión de los cónyuges existe también una cierta diferencia (13 de 21: 61,9\% vs. 8 de 21: $38,1 \% ; p=0,07)$. Los cónyuges de los pacientes con
TABLA IV. DISTRIBUCIÓN DE RASGOS Y TRASTORNOS DE PERSONALIDAD POR CÓNYUGE

\begin{tabular}{|c|c|c|c|}
\hline $\begin{array}{l}N .^{o} \text { total de } \\
\text { cónyuges de } \\
\text { pacientes con } \\
\text { dolor crónico }\end{array}$ & $\begin{array}{c}N .^{\circ} \text { de rasgos } \\
\text { y trastornos } \\
\text { personalidad } \\
\text { por cónyuge }\end{array}$ & $\begin{array}{c}N .^{o} \text { cónyuges } \\
\text { de pacientes } \\
\text { con dolor no } \\
F M\end{array}$ & $\begin{array}{c}N .^{o} \text { cónyuges } \\
\text { de pacientes } \\
\text { con dolor } \\
F M\end{array}$ \\
\hline $36(40 \%)$ & 1 & $9(30 \%)$ & $27(45 \%)$ \\
\hline 4 & 2 & 2 & 2 \\
\hline 6 & 3 & 2 & 4 \\
\hline 3 & 4 & 1 & 2 \\
\hline 4 & 5 & 2 & 2 \\
\hline 2 & 6 & 0 & 2 \\
\hline 1 & 7 & 0 & 1 \\
\hline 1 & 8 & 0 & 1 \\
\hline 0 & 9 & 0 & 0 \\
\hline 0 & 10 & 0 & 0 \\
\hline 0 & 11 & 0 & 0 \\
\hline 1 & 12 & 0 & 1 \\
\hline
\end{tabular}


dolor FM padecen más inestabilidad emocional y son más indecisos que los de pacientes con dolor no FM.

No se han encontrado asociaciones significativas entre las variables, excepto entre la inestabilidad emocional del cónyuge y el tiempo de convivencia en años $(\mathrm{p}<0,05)$ : hay más inestabilidad emocional en los cónyuges de pacientes con dolor crónico a partir de los 25 años de convivencia (17 de 38: 44,7 \% vs. 21 de 38: 55,3\%).

Existen relaciones estrechas entre trastorno de conversión del cónyuge y tiempo de convivencia, sin alcanzar significación estadística (9 de 19: 47,4 \% vs. 10 de 19: $52,6 \% ; p=0,07)$ : el trastorno de conversión de los cónyuges aumenta a partir de los 25 años de convivencia.

En los cónyuges de pacientes con FM se encuentran asociaciones específicas que no se han detectado entre los cónyuges de los pacientes con dolor crónico no FM (Tabla V).

\section{DISCUSIÓN}

En la investigación realizada se ha encontrado que existen tipos específicos de rasgos y trastornos de personalidad entre los cónyuges de pacientes con dolor crónico, así como que existen diferencias en relación al grupo control. Queda por dilucidar si los rasgos y trastornos de personalidad del cónyuge de paciente con dolor crónico influyen en el sufrimiento del paciente o viceversa.

El Manual de enfermedades psiquiátricas (DSM-IVTR) presenta los trastornos de personalidad en tres grupos, en los que hay superposiciones:

- Grupo A: trastornos de personalidad paranoide, esquizoide y esquizotípico.

- Grupo B: trastornos de personalidad antisocial, borderline, histriónico y narcisista.

- Grupo C: trastornos de personalidad evitativo o ansioso, dependiente, y obsesivo-compulsivo.

Hemos encontrado que el $42 \%$ de los cónyuges de pacientes con dolor crónico entran en el grupo B (personas emotivas, melodramáticas, imprevisibles) y el $34 \%$ en el $\mathrm{C}$ (personas con ansiedad o temor permanente), por lo que conviene repasar brevemente las características propias de algunos de estos trastornos.

- Paranoides: fríos y distantes en las relaciones sociales, sospechan continuamente de los demás, llevan consigo múltiples rencores.

- Histriónicos: teatrales, con una emotividad exagerada; reaccionan mal si no son el centro de atención; seductores o provocativos.

- Narcisistas: ideas irrealistas de grandiosidad; sentimientos de superioridad; creen ser especiales o únicos; se aprovechan de los demás para sus fines, caen en la envidia; carecen de empatía o capacidad de darse cuenta de qué ocurre a su alrededor y qué necesitan los demás.
TABLA V. ASOCIACIONES EN CÓNYUGES DE PACIENTES CON FM

\begin{tabular}{|c|c|c|c|}
\hline Factor & Trastorno & Probabilidad & Significación \\
\hline $\mathrm{CIF}>67$ & $\begin{array}{l}\text { Obsesiva } \\
n=11 ; 64,7 \% \\
\text { Obsesiva } \\
n=6 ; 35,3 \%\end{array}$ & $47 \%$ & $\mathrm{p}<0,001$ \\
\hline $\begin{array}{l}\text { Dolor paciente } \\
<16 \text { años } \\
\text { Dolor paciente } \\
>16 \text { años }\end{array}$ & $\begin{array}{l}\text { Inestabilidad } \\
\mathrm{n}=24 ; 70,6 \% \\
\text { Inestabilidad } \\
\mathrm{n}=10 ; 29,4 \%\end{array}$ & $41 \%$ & $p=0,065$ \\
\hline $\begin{array}{l}\text { Dolor paciente } \\
<16 \text { años } \\
\text { Dolor paciente } \\
>16 \text { años }\end{array}$ & $\begin{array}{l}\text { Indecisión } \\
\mathrm{n}=13 ; 100 \% \\
\text { Indecisión } \\
\mathrm{n}=0\end{array}$ & $99 \%$ & $\mathrm{p}<0,01$ \\
\hline $\begin{array}{l}\text { Total escala dolor } \\
>13 \\
\text { Total escala dolor } \\
<13\end{array}$ & $\begin{array}{l}\text { Inestabilidad } \\
\mathrm{n}=26 ; 76,5 \% \\
\text { Inestabilidad } \\
\mathrm{n}=8 ; 46,2 \%\end{array}$ & $45 \%$ & $\mathrm{p}<0,001$ \\
\hline $\begin{array}{l}\text { Total escala dolor } \\
>13 \\
\text { Total escala dolor } \\
<13\end{array}$ & $\begin{array}{l}\text { Indecisión } \\
\mathrm{n}=6 ; 100 \% \\
\text { Indecisión } \\
\mathrm{n}=7 ; 53,8 \%\end{array}$ & $86 \%$ & $\mathrm{p}<0,01$ \\
\hline $\begin{array}{l}\text { Convivencia } \\
>25 \text { años } \\
\text { Convivencia } \\
<25 \text { años }\end{array}$ & $\begin{array}{l}\text { Inestabilidad } \\
\mathrm{n}=20 ; 58,8 \% \\
\text { Inestabilidad } \\
\mathrm{n}=14 ; 41,2 \%\end{array}$ & $48 \%$ & $\mathrm{p}<0,001$ \\
\hline $\begin{array}{l}\text { Convivencia } \\
>25 \text { años } \\
\text { Convivencia } \\
<25 \text { años }\end{array}$ & $\begin{array}{l}\text { Indecisión } \\
\mathrm{n}=8 ; 61,5 \% \\
\text { Indecisión } \\
\mathrm{n}=5 ; 38,5 \%\end{array}$ & $45 \%$ & $\mathrm{p}<0,001$ \\
\hline Cónyuge enfermo & $\begin{array}{l}\text { Obsesiva } \\
\mathrm{n}=9 ; 52,9 \% \\
\text { Obsesiva } \\
\mathrm{n}=8 ; 47,1 \%\end{array}$ & $50 \%$ & $\mathrm{p}<0,001$ \\
\hline Cónyuge sano & $\begin{array}{l}\text { Introversión } \\
\mathrm{n}=29 ; 54,7 \%\end{array}$ & $87 \%$ & $\mathrm{p}<0,05$ \\
\hline Cónyuge enfermo & $\begin{array}{l}\text { Introversión } \\
\mathrm{n}=24 ; 45,3 \%\end{array}$ & & \\
\hline $\begin{array}{l}\text { Tiempo diagnóstico } \\
<7 \text { años } \\
\text { Tiempo diagnóstico } \\
>7 \text { años }\end{array}$ & $\begin{array}{l}\text { Obsesiva } \\
n=11 ; 64,7 \% \\
\text { Obsesiva } \\
n=6 ; 35,3 \%\end{array}$ & $14 \%$ & $\mathrm{p}<0,01$ \\
\hline
\end{tabular}

Probabilidad: OR/OR+1. 
- Evitativos o ansiosos: evitan cualquier situación de riesgo y los contactos interpersonales -aunque los desearían- por miedo al rechazo, a la vergüenza, al ridículo y a ser humillados; se ven personalmente inferiores a los demás; temen hablar en público o ser el centro de atención; sufren por el aislamiento.

- Obsesivo-compulsivos o anancásticos: son perfeccionistas, inflexibles y se preocupan excesivamente por el orden; odian los errores y les resulta difícil decidir por temor a equivocarse, lo que les llevaría a perder la estima y aprecio de los demás y reavivaría su profundo sentimiento de inferioridad; cuidan con exageración los detalles, las reglas, las listas de cosas que hay que hacer, que les hace sentirse útiles y valiosos; se dedican obsesivamente al trabajo; no tienen capacidad de delegar por desconfianza y necesidad de controlar todo; caen con frecuencia en escrúpulos.

La epidemiología en el campo de los trastornos de personalidad y, en general, en el campo de las enfermedades mentales está por desarrollar (14), aunque se conocen algunos datos. Los pacientes hiperfrecuentadores en Atención Primaria (15) muestran una elevada prevalencia de trastornos de la personalidad: compulsivo $(56,7 \%)$, esquizoide $(46,7 \%)$, dependiente $(45 \%)$, paranoide $(18 \%)$, esquizotípico $(8 \%)$ y bordeline (4\%).

En un reciente estudio (16) acerca de la prevalencia de desórdenes mentales en las prisiones españolas se afirma que es del 41,2 \%; cuatro años antes, un informe interno de la Dirección General de Instituciones Penitenciarias la situaba en el $46 \%$. En un estudio publicado últimamente los autores (17) hallan que la prevalencia del trastorno obsesivo-compulsivo era de 7,8 \% en Estados Unidos. No hemos encontrado estudios de este tipo en la población española.

En la tabla IV se aprecia que el $40 \%$ de los cónyuges de pacientes con dolor crónico en nuestro estudio tienen trastornos de personalidad (45\% en los cónyuges de pacientes con FM; $30 \%$ en los cónyuges de pacientes con dolor crónico no FM). Esta prevalencia se acerca muy estrechamente a la que se encuentra en los internos de las prisiones españolas. No parece existir similitud en relación a los pacientes hiperfrecuentadores en Atención Primaria. Sin embargo, hemos encontrado claras diferencias en la prevalencia de rasgos y trastornos de personalidad entre los cónyuges de pacientes con FM y con dolor crónico no FM, resultando con frecuencia que es el doble en el caso de los cónyuges de pacientes con FM. Una circunstancia que podría sesgar estos resultados es el diferente tamaño muestral de ambos grupos.

Los pacientes con FM tienen una puntuación más alta en la escala total del dolor que los pacientes con dolor crónico no FM, lo que puede explicarse por el fenómeno de la sensibilización central (6). En términos generales, a partir de los 25 años de convivencia hay más inestabilidad emocional en los cónyuges y es más frecuente en ellos el trastorno de conversión. Por otro lado, los cónyuges de los pacientes con dolor crónico FM tienen más inestabilidad emocional y son más indecisos que los de pacientes con dolor no FM.

El trastorno obsesivo es más frecuente en los cónyuges de los pacientes con FM cuando la puntuación CIF es menor de 67, en los primeros 7 años tras el diagnóstico de la FM o si el cónyuge no presenta trastornos físicos, aunque la diferencia clínicamente es escasa. Parece ser más prevalente, por tanto, en los primeros años de la enfermedad. La explicación podría deberse a la aparición de dificultades en la relación de pareja que quedan al descubierto al iniciarse los síntomas de la enfermedad que exigen un cambio de posición e involucración del cónyuge y los familiares cercanos.

Los cónyuges de los pacientes con FM presentan más inestabilidad emocional en los primeros 16 años de dolor del paciente, cuando el total de la escala del dolor es superior a 13 o a partir de los 25 años de convivencia. Por tanto, parece ser más prevalente en una fase más avanzada de la enfermedad. El cónyuge asume la enfermedad de su pareja, intentando demostrar que es más fuerte, por lo que, con cierta frecuencia, se presenta sumiso, complaciente, siendo consciente de que al no tener dolor está en condiciones muy superiores al paciente; ese esfuerzo puede ser la causa de la desestabilización emocional. Suelen presentarse como tímidos, aunque buenos acompañantes. La hiperresponsabilidad que manifiestan en su historia vital pasada los pacientes con FM, junto a su actual debilidad e impotencia ante los acontecimientos vitales, puede haber condicionado una personalidad sumisa en su cónyuge.

El rasgo indecisión es más prevalente en los cónyuges de pacientes con FM en los primeros 16 años de dolor del paciente, si el total de la escala del dolor es inferior a $13 \mathrm{o}$ a partir de los 25 años de convivencia. Este hallazgo tiene una difícil explicación.

Curiosamente, se muestran más introvertidos los cónyuges sanos de pacientes con FM que los que manifiestan alguna enfermedad de tipo físico. Pensamos que este rasgo podría acentuarse por su inexperiencia ante la enfermedad y que podría influir un cierto miedo y perplejidad ante los síntomas de la FM que observan.

Sería deseable poder aplicar un método rápido, sencillo y fiable de detección de los rasgos y especialmente de los trastornos de personalidad de los cónyuges de pacientes con dolor crónico, principalmente con FM, con el fin de intentar evitar el deterioro de la convivencia familiar de estos pacientes, debido a la importancia que tiene el entorno (7) en que se mueve para una mejor recuperación del paciente. Pensamos que el Cuestionario Multifactorial de Personalidad HERAS (10) es un buen instrumento en este sentido y que, a los cónyuges con trastornos de personalidad, convendría explicarles el problema y derivarlos al psicólogo. 
Nos proponemos repetir los test al cabo de unos años para valorar los cambios de rasgos y trastornos de personalidad en los cónyuges, detectar el inicio de una enfermedad mental, e intentar dilucidar si los rasgos y trastornos de personalidad del cónyuge influyen en el sufrimiento del paciente o viceversa.

\section{CORRESPONDENCIA:}

Eliseo Collazo

Clínica del Dolor

Hospital Universitario Reina Sofía

Avda. Menéndez Pidal, s/n

14004 Córdoba

e-mail: ecollazo@comcordoba.com

\section{BIBLIOGRAFÍA}

1. Bosch F, Toranzo I, Baños JE. A survey of pain complaints and treatment by general practitioners in the Spanish public health organization. Clin J Pain 1990;6:206-11.

2. Mäntyselkä P, Kumpusalo E, Ahonen R, Kumpusalo A, Kauhanen J, Viinamäki H, et al. Pain as a reason to visit the doctor: A study in Finnish primary health care. Pain 2001;89:175-80.

3. Montoya P, Larbig W, Braun C, Preissl H, Birbaumer N. Influence of social support and emotional context on pain processing and magnetic brain responses in fibromyalgia. Arthritis Rheum 2004;50:4035-44.

4. Goodenow C, Reisine S T, Grady KE. Quality of social support and associated social and psychological functioning in women with rheumatoid arthritis. Health Psychol 1990;9:266-84.

5. Savelkoul M, Post MWM, De Witte L P, et al. Social support, coping and subjective well-being in patients with rheumatic diseases. Patient Educ Couns 2000;39:205-18.
6. Rotés-Querol J. La fibromialgia en el año 2002. Rev Esp Reumatol 2005;32:77-81.

7. Azkue JJ, Bilbao J, Montoya P, Aira Z, Buesa I, Salgueiro M. Valoración de factores sociales y clínicos en el síndrome de fibromialgia. Rev Soc Esp Dolor 2009;6:323-9.

8. Martínez E, González O, Crespo JM. Fibromialgia: definición, aspectos clínicos, psicológicos, psiquiátricos y terapéuticos. Salud mental. Aten Primaria 2003;4:2-7.

9. Camino Vallhonrat A, Jiménez Rico B, de Castro-Palomino i Serra M, Fábregas M. Ira, fibromialgia y ansiedad: aproximación terapéutica desde un CSM. Rev Asoc Esp Neuropsiq 2009;29(103):203-15.

10. Disponible en: http://www.psicosomatica.net/PacientesYUsuarios/8.CUESTIONARIO\%20MULTIFAC....pdf.

11. Pinillos JL. Cuestionario de Personalidad CEP. Madrid: TEA Ediciones, S.A.; 1971.

12. Esteve-Vives J, Rivera Redondo J, Salvat Salvat M, et al. Propuesta de una versión de consenso del Fibromialgia Impact Questionnaire (FIQ) para la población española. Reumatol Clin 2007;3(1):21-4.

13. Fernández E. Estudios epidemiológicos (STROBE). Med Clin 2005;125(Supl. 1):43-8.

14. Srinivasa Murthy R, Lakshminarayana R. Is it possible to carry out high-quality epidemiological research in psychiatry with limited resources? Curr Opin Psychiatry 2005;18:565-71.

15. Rodríguez Muñoz C, Cebrià Andreu J, Corbella Santomà $S$, Segura Bernal J, Sobreques Soriano J. Rasgos de personalidad y malestar psíquico asociados a los pacientes hiperfrecuentadores de consultas de Atención Primaria. Medifam 2003;13:143-50.

16. Vicens E, Tort V, Dueñas RM, Muro Á, Pérez-Arnau F, Arroyo JM, Et al. The prevalence of mental disorders in Spanish prisons. Crim Behav Ment Health 2011;21(5):321-32.

17. Grant JE, Mooney ME; Kushner MG. Prevalence, correlates, and comorbidity of DSM-IV obsessive-compulsive personality disorder: Results from the National Epidemiologic Survey on Alcohol and Related Conditions. J Psychiatr Res 2012;46(4):469-75. 